\title{
The who and how of attentional bias in cannabis users: associations with use severity, craving, and interference control
}

\author{
Emese Kroon ${ }^{1,2}$, Lauren Kuhns ${ }^{1,2}$, Annette Dunkerbeck ${ }^{3}$ \& Janna Cousijn ${ }^{1,2,4}$ \\ ${ }^{1}$ Neuroscience of Addiction (NofA) Lab, Department of Psychology, University of Amsterdam, The Netherlands \\ ${ }^{2}$ ADAPT-laboratory, Department of Psychology, University of Amsterdam, the Netherlands \\ ${ }^{3}$ Dimence, SCOS, Deventer \\ ${ }^{4}$ Department of Psychology, Education \& Child Studies, Erasmus University Rotterdam, The Netherlands
}

Correspondence: Emese Kroon, e.kroon@uva.nl, P.O. box 15916, 1001 NK Amsterdam, The Netherlands

Declarations of interest: none

Funding: This research was supported by grant 1R01 DA042490-01A1 awarded to Janna Cousijn from the National Institute on Drug Abuse/National Institute of Health.

Author contributions: All authors reviewed and approved the final version. Conceptualization: EK, LK \& JC; Methodology: EK, LK \& JC; Investigation: EK, LK, AD \& JC; Formal Analysis: EK \& LK; Data curation: EK \& LK; Writing - Original draft: EK; Writing - Review \& Editing: LK, AD \& JC; Visualization: EK; Supervision: JC; Funding Acquisition: JC.

Data Accessibility: The data, code and materials of this study are available from the corresponding author upon reasonable request.

Preregistration: The analysis plans were preregistered (https://aspredicted.org/7JT_TN7)

Preprint: this is a preprint version of this article which has not been peer-reviewed or published 


\section{Abstract}

Background and Aim. Cognitive and motivational processes are thought to underlie cannabis use disorder (CUD), but research assessing how cognitive processes (e.g. interference control (IC)) interact with implicit motivational processes (e.g. attentional bias (AB)) and explicit motivation (i.e. craving) is lacking. We assessed the presence of $A B$ in cannabis users with varying use severity and tested models of moderation, mediation, and moderated mediation to assess how AB, craving, and IC interact in their association with measures of cannabis use. Design. Cross-sectional. Setting and Participants. Eight studies performed in the Netherlands including never-sporadic, occasional $(\leq 1 /$ month), and regular cannabis users ( $\geq 2 /$ week), and individuals in treatment for CUD were combined ( $N=560 ; 71 \%$ male). Measurements. All studies included a Classic Stroop task to assess IC, a Cannabis Stroop task to assess $A B$, and a pre- and post-session measure of craving. Both heaviness of cannabis use (grams per week) and severity of use related problems (CUDIT-R scores) were included. Findings. Only those in treatment for CUD showed an $A B$ and group differences in $A B$ were only observed when comparing those in treatment with never to sporadic users. Looking at occasional-regular users, IC was negatively associated with heaviness, but not severity of use. There was no direct association between $A B$ and measures of cannabis use. However, average craving during the session, but not session induced craving, mediated this association between $A B$ and heaviness as well as severity of use; higher $A B$ was associated to heavier use and more severe problems through increased craving. Conclusions. $A B$ was only present in cannabis users with the most severe problems and craving mediated the association between $A B$ and both heaviness and severity of use in occasional to regular users. Furthermore, the association of IC with heaviness but not severity of use may point to sub-acute effects.

Key words: attentional bias, craving, interference control, cannabis use, cognition, motivation 


\section{INTRODUCTION}

Excessive cannabis use and cannabis use disorder (CUD) are considered major health problems. Trends in cannabis legalisation, increasing potency and decreasing harm perceptions (1) highlight the urgency of research into the mechanisms underlying CUD. Traditional theories of addiction propose central roles for both cognitive and motivational processes (2), but research assessing both cognitive and motivational processes and their interactions in cannabis users is lacking.

Increased salience of substance-related cues in substance users is thought to bias behaviour towards substance use, which can present itself as a cue-induced attentional bias (AB) and craving (3). These drug-oriented motivational processes may more easily result in actual substance use in individuals with relatively limited cognitive control $(4,5)$. The classical Stroop task has been used to measure cognitive control (6), in which slower responses on incongruent trials, controlled for congruent trials, are an indication of lower IC. Modified drug Stroop tasks have been developed (e.g. 7) and the extent to which substance-related (e.g. weed or blunt) relative to matched neutral words (e.g. floor or table) slow down color naming is taken as an index of $A B$, which is expected to relate to substance use history (8).

Several studies investigated the role of IC, AB and craving in cannabis use and CUD. One study using the classical Stroop to measure IC found poorer IC and altered brain activity in weekly to daily users relative to non-sporadic using controls when responding to incongruent trials (9). However, others, found no performance differences when comparing near-daily users and controls (e.g. 10) or only found differences in task-related brain activity in at-risk and treatment samples (e.g. 11-13). Similarly, $A B$ has been identified in cannabis users ranging from lifetime users to those in treatment for CUD (14-16), while others do not observe AB using a Cannabis Stroop even in near daily users and those in treatment for CUD (17-19). Craving, however, has consistently been associated with heavier use (20; review) and has been shown to be predictive of cannabis use and related problems six months later (14). Also, craving has been association with both $A B(21$; meta-analysis) and IC (e.g. 16) in studies using the Cannabis and Classical Stroop. 
These mixed findings could in part be explained by the differential role that $A B$, craving and IC play across trajectories of cannabis use towards CUD. IC may be lower and $A B$ and craving may be higher in heavier and dependent users $(19,20,22-25)$. Unfortunately, most studies look at these constructs separately and have a limited range of cannabis use severity included in the sample. It remains unclear which cannabis users have an $A B$ and how this relates to craving and IC (e.g. 14). Furthermore, a meta-analysis by Field et al. (2009) found a small but significant association between $A B$ and craving in substance users, indicating that previous studies lack power to detect such small effects (21). To overcome these problems and systematically assess the potential interactions between cognitive and motivational processes in a large sample of cannabis users with variable use frequency, this study combines eight studies conducted in our lab that included a pencil and paper version of the Classical Stroop and the Cannabis Stroop, and assessments of craving.

First, focussing on $A B$, we will assess whether groups of never-sporadic users, occasional users, regular users and those in treatment for CUD show an $A B$ towards cannabis cues and whether $A B$ differs between these groups. We expect an $A B$ in regular users and those in treatment for CUD only, that differs from the never- and sporadic users (20; review). In occasional to regular users, excluding the CUD group to avoid effects of recent cessation on the outcomes, we will assess whether $A B$, craving, IC, heaviness of current use, and severity of cannabis use-related problems are indeed associated with each other in this broad range of users.

Second, we will assess how the cannabis $A B$, craving and IC interact in their association with heaviness and severity of cannabis use. We will test different theory informed models; we will assess whether $A B$, craving and/or IC are predictive of heaviness of cannabis use and/or severity of cannabis use-related problems (Figure 1A; e.g 26). Then we will assess the proposed moderating role of cognitive processes in overcoming motivational urges (Figure $1 B$ \& Figure $1 C ;, 4,5$ ). AB could increase craving or vice versa, subsequently leading to increased cannabis use or use-related problems (e.g. $3,27)$. Therefore, we will also separately assess whether $A B$ or craving act as mediators in the association between the other variable with heaviness of use and severity of cannabis-use-related 
problems (Figure 1D \& Figure 1E). Then, to combine these moderation and mediation models, we will assess whether IC moderates the association of craving and/or AB with heaviness/severity cannabis use in the proposed mediation models (Figure $1 \mathrm{~F} \&$ figure $1 \mathrm{G}$ ).

\section{METHODS}

We combined data from eight studies $(19,28,29,30$, four studies unpublished) that included similar measures of $A B, I C$ and craving. In all studies, procedures were approved by the ethics committee of the corresponding department and all participants were fully informed and provided informed consent before the start of the experiment.

\section{Participants}

Individuals with known color-blindness $(\mathrm{N}=2)$ and those that tested positive on drugs other than cannabis during the test session $(\mathrm{N}=7)$ were excluded from the analyses, resulting in a total sample of 560 participants ( $71 \%$ male).

\section{Materials}

Assessments

Participants reported age, gender, weekly cannabis use in grams (heaviness of use) and completed the cannabis use disorder identification test-revised (CUDIT-R; 31 ) to assess severity of cannabis use-related problems. Smoking (yes/no), the Fagerström test for Nicotine dependence (FTND; 32) and the alcohol use disorder identification test (AUDIT; 33) were included to assess the severity of drug use other than cannabis.

Craving

Craving was assessed at the start and end of the session using a visual analogue scale or the marijuana craving questionnaire (MCQ; Figure S1; ,34). Session induced craving (start - end score) and average craving were calculated, after which scores were standardized.

Classical Stroop: interference control

The Classical Stroop task included three different cards that were presented in a fixed order $(6,35)$. All cards included ten rows of ten words/blocks which participants were instructed to read over 
row-by-row, as fast as possible, according to the card instructions. First, participants were instructed to read the words red, green, blue, and yellow as printed in black (word card). Second, participants were instructed to name the color of the color blocks (color card). Last, participants were instructed to name the incongruent color in which the words red, green, blue, and yellow were printed (colorword card). Reaction times were recorded and IC scores were calculated using this formula: reaction time color-word card/((reaction time word card + reaction time color card)/2) (36). Higher scores indicated lower IC.

\section{Cannabis Stroop: attentional bias}

The Cannabis Stroop task included two different cards presented in counterbalanced order (29). Each card included eight rows of seven words that were all printed in red, green, blue or yellow. The words on both cards were matched on word length and number of syllables, but on one card the words were neutral (e.g. poster), while the words on the other card were cannabis-related (e.g. stoned). Participants were instructed to name the color in which each word was printed, row by row, from left to right, as fast as they could. A stopwatch was used to record the time needed to complete each card. $A B$ scores were calculated using the following formula: reaction time cannabis card reaction time neutral card, with higher scores being indicative of a relatively higher bias for cannabis words.

\section{Procedures}

While exact procedures and the order of included measures varied between studies (Figure S1), the Cannabis Stroop was always completed before the Classical Stroop. Also, cannabis-related questionnaires, aside from the pre-session craving, were always completed after the Stroop tasks.

\section{Data Analysis}

Grouping \& exclusion

Participants were classified as never-sporadic user (no lifetime or no use in the last year), occasional users (maximum of once per month during the last year), regular users (minimum of twice per week during the last year) or CUD (in treatment at the moment of testing; Table 1) using the first 
question of the CUDIT-R (31) (note: in study 8, grouping was based on self-reported last year use) and treatment status. Individuals that did not fit any of these groups ( $N=8)$ were excluded (Table S1). IC, $A B$, craving, grams/week of use and CUDIT-R scores that were more than 3 standard deviations from the mean were excluded to reduce potential effects of outliers.

\section{Attentional bias}

One-sample t-tests were run to assess whether there was an $A B$ to cannabis words (whether the $A B$ was different from zero) per group. Then, an ANOVA was performed to assess group differences in $A B$, with post-hoc independent sample t-tests to explore the differences. Then, in all occasional to regular users, correlation analyses were conducted to assess how heaviness of cannabis use (grams/week), severity of cannabis use-related problems (CUDIT-R score), AB, IC, and session induced (SI) craving were associated with each. Analyses were run in JASP (version 0.14.1.0; ,37).

Attentional bias, interference control and craving: their association with cannabis use

Only current occasional to regular users were included in the following analyses (Table 1; excluding CUD group due to potential effects of recent cessation). Simple and multiple regression analyses were conducted to assess whether $A B, I C$, and/or SI craving were predictive of heaviness of cannabis use and severity of cannabis use-related problems (Figure 1A). Moderation analyses were conducted to assess whether IC moderates the association between $\mathrm{SI}$ craving (Figure 1B) or AB (Figure 1C) and heaviness of cannabis use and severity of cannabis use-related problems. Then, to assess the proposed relation between $A B$ and $S I$ craving in their association with cannabis use outcomes, we ran a mediation analysis to see whether $A B$ mediates the association between SI craving and heaviness of cannabis use or severity of cannabis use related problems (Figure 1D) or the reverse (Figure 1E, Figure S3A). Combining this, moderated-mediation analyses were run to assess whether IC moderates the association between SI craving and $A B$ with heaviness of cannabis use or cannabis use related problems in the proposed mediation models (Figure 1F \& Figure 1G, Figure S3B). All included variables were mean centered. Analyses were run in $\mathrm{R}$ (version $4.1 .2 ; 38$ ) creating the models (Figure B-G) using the processR (version 0.2.6; 39) package and running them in lavaan (version 0.6-9; 40) 
using maximum likelihood estimation. Exclusion criteria, grouping variables and analyses were preregistered (https://aspredicted.org/7JT TN7). Exploratory analyses were reported as such and multiple comparison corrections were applied in analyses that were not pre-registered.

\section{RESULTS}

\section{Sample characteristics}

Groups significantly differed on all variables (see Table 1). Exploratory independent sample ttests showed varying patterns of differences for all variables with a general tendency of more severe alcohol, cigarette use, and more limited IC in more severe cannabis users and no differences between never-sporadic users and occasional users. Notably, session induced craving was only positive in regular users.

\section{Group differences in attentional bias \& correlations between variables}

Only the CUD group showed an $A B(t(92)=2.39, p=.019, d=.25)$. The never-sporadic $(t(96)$ $=1.31, p=.192, d=.13)$, occasional $(t(34)=0.38, p=.704, d=.07)$, and regular users $(t(319)=1.72, p$ $=.087, d=.10)$ did not show this $\mathrm{AB}$. AB differed between groups $\left(F(3,541)=3.1, p=.026, \eta^{2}=.017\right.$; Table 1), with post-hoc analyses revealing a higher bias in CUD (and regular users at $p=.050$ ) versus never-sporadic users (Figure 2). Exploratory sensitivity analyses adding variables that differed between groups (Table 1) showed that the effect was independent of age and IC but no longer significant after correction for AUDIT and FTND.

Focussing on occasional and regular users, correlational analysis revealed a positive association between heaviness of cannabis use (Gram/week) and severity of cannabis use (CUDIT-R scores; $\left.r_{s}(347)=.49, p<.001\right)$. CUDIT-R score was not associated with any of the other variables (highest $r_{s}=.10$, with $p=.06$ ), but gram/week was positively associated with Classical Stroop scores $\left(r_{s}(343)=.20, p<.001\right)$, indicating worse IC in more severe users. No other correlations between IC, craving and $\mathrm{AB}$ were observed (highest $r_{s}=.08$, with $p=.16$ ). 


\section{Attentional bias, interference control and craving: their association with cannabis use}

In line with the correlational results, simple regression analyses (Figure $1 \mathrm{~A}$ ) showed and association between poorer IC and gram/week $\left(R^{2}=.037, F(1,343)=14.23, \beta=.015, \beta_{\mathrm{SE}}=.004, t=\right.$ 3.772, $p<.001)$, but not CUDIT-R score $\left(R^{2}=-.003, F(1,350)=.023, \beta=.004, \beta_{\mathrm{SE}}=.027, t=.150, p=\right.$ .881). AB and craving did not directly predict gram/week or CUDIT-R score (Table S2; Figure S2).

Moderation (Figure 1B \& Figure 1C; Table S3), mediation (Figure 1D \& Figure 1E; Table S4) and moderated-mediation (Figure 1F \& Figure 1G; Table S5) models revealed no other associations than the consistently present direct association between IC and gram/week (Figure S2).

\section{Exploratory analyses: the role of average session craving}

As session induced changes in craving do not necessarily reflect absolute feelings of craving, but rather to what extent the session affected craving in the individual, we re-ran the correlations, simple regressions, moderation, mediation, and moderated-mediation models with average session (AS) craving instead of session induced craving.

Correlational and simple regression analyses showed that AS craving was positively associated with gram/week $\left(r_{s}(330)=.30, p<.001 ; R^{2}=.057, F(1,330)=20.93, \beta=.977, \beta_{\mathrm{SE}}=.214, t=4.575, p<\right.$ $.001)$ and CUDIT-R $\left(r_{s}(338)=.26, p<.001 ; R^{2}=.074, F(1,338)=28.19, \beta=1.75, \beta_{\mathrm{SE}}=.331, t=5.309, p\right.$ $<.001)$ of use. Furthermore, higher AS craving during the session was associated with higher $A B$ $\left(r_{s}(336)=.15, p=.006\right)$ and lower IC (i.e. higher Stroop score; $\left.r_{s}(333)=.18, p=.001\right)$.

Moderation analyses revealed similar associations, also including the association between IC and heaviness of use (Table S7). However, mediation analyses revealed that AS craving mediated the association between $A B$ and both gram/week (indirect effect: $\beta=.050, \beta_{\mathrm{SE}}=.020, z=2.556, p=.011$ ) and CUDIT-R score (indirect effect: $\beta=.083, \beta_{\mathrm{SE}}=.032, z=2.602, p=.009 ;$ Table S7). These mediations were stable across the moderated-mediation models (CUDIT-R - indirect effect: $\beta=.089, \beta_{\text {SE }}=.033, z$ $=2.655, p=.008 ;$ gram $/$ week - indirect effect: $\left.\beta=.049, \beta_{\mathrm{SE}}=.019, z=2.552, p=.011\right)$, but IC did not act as a moderator but rather was directly associated with gram/week only (Table S7; Figure 4). 


\section{DISCUSSION}

We assessed the presence of $A B$ in cannabis users with different levels of use and how $A B$ interacted with craving and IC in its relationship with heaviness and severity of cannabis use. A clear strength of this study is the inclusion of a large sample with a large range of cannabis use severity ( $\mathrm{N}$ $=560$ ). Only those users in treatment for CUD showed an AB (significantly $>0$ ), which was significantly higher compared to never-sporadic users, but not compared to occasional and regular users. Poorer IC was consistently associated with heavier cannabis use, but not the severity of use related problems. However, in contrast to our hypotheses, IC did not moderate the association between AB and craving in their association with measures of cannabis use. Moreover, session induced craving did not mediate the association between $A B$ (nor vice versa) and measures of cannabis use, yet results changed when using average craving instead; craving mediated the association between $A B$ and heaviness as well as severity of use.

Our results suggest that $A B$ may be a clinical marker of CUD severity, while IC may generally be poorer in heavier users regardless of CUD problem severity. However, the associations between $A B$, craving $I C$ and cannabis use are complex. Looking at Figure $1, A B$ appears higher in more frequent users, but $A B$ did not directly relate to our measures of cannabis use (also not when exploratively including the CUD group in the regression analysis). It only did through its positive association with craving; those with higher $A B$ might have higher, potentially more 'trait-like' levels of craving, triggering a higher general likelihood to use. Most studies indicate that the relationship between craving and $A B$ is likely reciprocal (3), however, our results in which $A B$ affects use through craving are in line with earlier research in alcohol users in which training to increase $A B$ resulted in increased craving and subsequent use (41). The indirect effects of $A B$ via craving could also explain why some studies did not find direct associations between $A B$ and measures of use (e.g. 25, alcohol Stroop; 42, cocaine Stroop). However, our findings are cross-sectional and were only significant for average craving, not session induced craving. Studies investigating the temporal dynamics between $A B$ and craving are needed to further investigate this. 
The specific presence of $A B$ in the treatment (most severe) group, could explain some of the null findings of previous studies (e.g. 43) and could indicate its potential value as a clinical marker. However, research evaluating the relevance of assessing $A B$ for other substance use disorders in clinical settings are inconsistent (e.g. 27,44$)$ - while some studies show $A B$ to be associated with worse treatment outcomes or increased relapse rate $(45$, heroin; 46 , cocaine; 47 , alcohol) this is not $(45$, cocaine; 48 , tobacco) the case in all studies - and studies on the value of $A B$ as a marker of CUD severity and treatment outcomes are largely lacking. Hence, further research is required to systematically assess the clinical relevance of $A B$ to cannabis cues in clinical and non-clinical samples of cannabis users.

It must be noted that the group differences disappeared when controlling for AUDIT and FTND. Poly substance use is very common (49) and the higher use of alcohol and tobacco might arise from the same underlying factors as their heavy cannabis use. Including AUDIT and FTND as covariates is suboptimal for it likely deletes cannabis use-relevant variance. Furthermore, it seems theoretically unlikely that alcohol and tobacco use directly affect $A B$ for cannabis words, but further research with samples (more closely) matched on these variables are needed to confirm this.

Partially in line with our expectations, we consistently found lower IC to be associated with heavier cannabis use (small-medium effect; $r_{s}=.20$ ). While it is often argued that this could indicate of a lack of control over use $(4,5)$, the lack of association with severity of cannabis use related problems and the lack of interactions with $A B$ and craving may indicate that this association is a result of current heaviness of use and the associated sub-acute effects. Some earlier studies also failed to find a moderating role if IC (e.g. 19,24). Cannabis intoxication has been found to negatively affect Stroop performance (e.g. 50) and there is evidence that several cognitive functions recover with increased abstinence (e.g. 51). In line with this, an exploratory check in the CUD group, of which the majority have been abstinent for multiple days ( $53 \%$ at least 7 days of abstinence), showed that there is no association between IC and heaviness of use in the CUD group (Table S9). Further research is needed to assess (sub)acute effects and the potential for recovery. 
Several limitations of this study should be noted. While combining different studies increases the sample size and allows for more complex models to be tested, it also introduces differences in methodology between studies. This could have affected the results of session induced craving as sessions in all studies had different length and content aside from the measures we included. However, the difference in the results when including two different measures of craving (session induced vs. average craving during the session) highlight the importance of the exact measures included and the potential incomparability of those measures. Furthermore, it must be investigated whether our results generalize to other measures of cognitive functioning and $A B$ and whether these effects generalize to more ecologically valid situations in which $A B$ could affect craving and cannabis use.

Our results show that $A B$ as measured by the Cannabis Stroop was only present in those cannabis users with the most severe problems but that, even in less severe cannabis users, the size of the $A B$ was associated with higher craving and in turn higher cannabis use and related problems. While systematic research into the clinical relevance of these associations is crucial, these results highlight the potential importance of $A B$ in both heaviness and severity of cannabis use as well as the mechanisms by which $A B$ through increased craving could affect efforts to reduce or stop using cannabis. 


\section{REFERENCES}

1. UNODC. Drug Market Trends : Cannabis Opioids. World Drug Report 2021. 2021.

2. Bickel WK, Mellis AM, Snider SE, Athamneh LN, Stein JS, Pope DA. 21st century neurobehavioral theories of decision making in addiction: Review and evaluation. Pharmacology Biochemistry and Behavior. 2018;164(September 2017):4-21.

3. Field M, Cox WM. Attentional bias in addictive behaviors: A review of its development, causes, and consequences. Drug and Alcohol Dependence. 2008;97(1-2):1-20.

4. Robinson TE, Berridge KC. The incentive sensitization theory of addiction: Some current issues. Philosophical Transactions of the Royal Society B: Biological Sciences. 2008;363(1507):3137-46.

5. Hester R, Luijten M. Neural correlates of attentional bias in addiction. CNS Spectrums. 2014;19(3):231-8.

6. Stroop RJ. Studies of Interference in Serial Verbal Reactions. Journal of Experimental Psychology. 1935;121(1):15-23.

7. Ataya AF, Adams S, Mullings E, Cooper RM, Attwood AS, Munafò MR. Internal reliability of measures of substance-related cognitive bias. Drug and Alcohol Dependence. 2012;121(12):148-51.

8. Smith DG, Ersche KD. Using a drug-word Stroop task to differentiate recreational from dependent drug use. CNS Spectrums. 2014;19(3):247-55.

9. Battisti RA, Roodenrys S, Johnstone SJ, Pesa N, Hermens DF, Solowij N. Chronic cannabis users show altered neurophysiological functioning on Stroop task conflict resolution. Psychopharmacology. 2010;212(4):613-24. 
10. Takagi M, Lubman DI, Cotton S, Fornito A, Baliz Y, Tucker A, et al. Executive control among adolescent inhalant and cannabis users. Drug and Alcohol Review. 2011;30(6):629-37.

11. Banich MT, Crowley TJ, Thompson LL, Jacobson BL, Liu X, Raymond KM, et al. Brain activation during the Stroop task in adolescents with severe substance and conduct problems: A pilot study. Drug and Alcohol Dependence. 2007;90(2-3):175-82.

12. Kober H, Devito EE, Deleone CM, Carroll KM, Potenza MN. Cannabis abstinence during treatment and one-year follow-up: Relationship to neural activity in men. Neuropsychopharmacology. 2014;39(10):2288-98.

13. Thayer RE, Feldstein Ewing SW, Dodd AB, Hansen NS, Mayer AR, Ling JM, et al. Functional activation during the Stroop is associated with recent alcohol but not marijuana use among high-risk youth. Psychiatry Research - Neuroimaging. 2015;234(1):130-6.

14. Cousijn J, van Benthem P, van der Schee E, Spijkerman R. Motivational and control mechanisms underlying adolescent cannabis use disorders: A prospective study. Developmental Cognitive Neuroscience. 2015;16:36-45.

15. Cane JE, Sharma D, Albery IP. The addiction Stroop task: Examining the fast and slow effects of smoking and marijuana-related cues. Journal of Psychopharmacology. 2009;23(5):510-9.

16. Cousijn J, Watson P, Koenders L, Vingerhoets WAM, Goudriaan AE, Wiers RW. Cannabis dependence, cognitive control and attentional bias for cannabis words. Addictive Behaviors. $2013 ; 38(12): 2825-32$.

17. Carpenter KM, Schreiber E, Church S, McDowell D. Drug Stroop performance: Relationships with primary substance of use and treatment outcome in a drug-dependent outpatient sample. Addictive Behaviors. 2006;31(1):174-81.

18. Asmaro D, Carolan PL, Liotti M. Electrophysiological evidence of early attentional bias to drug-related pictures in chronic cannabis users. Addictive Behaviors. 2014;39(1):114-21. 
19. Van Kampen AD, Cousijn J, Engel C, Rinck M, Dijkstra BAG. Attentional bias, craving and cannabis use in an inpatient sample of adolescents and young adults diagnosed with cannabis use disorder: The moderating role of cognitive control. Addictive Behaviors. 2020;100(September 2019):106126.

20. Kroon E, Kuhns L, Hoch E, Cousijn J. Heavy cannabis use, dependence and the brain: a clinical perspective. Addiction. 2019;

21. Field M. A Meta-Analytic Investigation of the Relationship Between Attentional Bias and Subjective Craving in Substance Abuse. Psychological Bulletin. 2009;135(4):589-607.

22. Marhe R, Waters AJ, Van De Wetering BJM, Franken IHA. Implicit and explicit drug-related cognitions during detoxification treatment are associated with drug relapse: An ecological momentary assessment study. Journal of Consulting and Clinical Psychology. 2013;81(1):112.

23. Waters AJ, Marhe R, Franken IHA. Temptations To Use Heroin and Cocaine. 2015;219(3):90921.

24. Cousijn J, Watson P, Koenders L, Vingerhoets WAM, Goudriaan AE, Wiers RW. Cannabis dependence, cognitive control and attentional bias for cannabis words. Addictive Behaviors [Internet]. 2013;38(12):2825-32. Available from: http://dx.doi.org/10.1016/j.addbeh.2013.08.011

25. Hallgren K, McCrady B. Interference in the alcohol Stroop task with college student binge drinkers. Journal of Behavioral Health. 2013;2(2):112.

26. Kroon E, Kuhns L, Cousijn J. The short-term and long-term effects of cannabis on cognition: recent advances in the field. Current Opinion in Psychology. 2021;38:49-55.

27. Field M, Marhe R, Franken IHA. The clinical relevance of attentional bias in substance use disorders. CNS Spectrums. 2014;19(3):225-30. 
28. Cousijn J, Snoek RWM, Wiers RW. Cannabis intoxication inhibits avoidance action tendencies: A field study in the Amsterdam coffee shops. Psychopharmacology. 2013;229(1):167-76.

29. Cousijn J, van Benthem P, van der Schee E, Spijkerman R. Motivational and control mechanisms underlying adolescent cannabis use disorders: A prospective study. Developmental Cognitive Neuroscience. 2015;16:36-45.

30. Cousijn J, van Duijvenvoorde ACK. Cognitive and mental health predictors of withdrawal severity during an active attempt to cut down cannabis use. Frontiers in Psychiatry. 2018;9:110.

31. Adamson SJ, Kay-Lambkin FJ, Baker AL, Lewin TJ, Thornton L, Kelly BJ, et al. An improved brief measure of cannabis misuse: The Cannabis Use Disorders Identification Test-Revised (CUDITR). Drug and Alcohol Dependence. 2010;110(1-2):137-43.

32. Heatherton T, Kozlowski L, Frecker R, Fagerström K. The Fagerström Test for Nicotine Dependence: a revision of the Fagerström Tolerance Questionnaire. Vol. 86, British Journal of Addiction. 1991. p. 1119-27.

33. Saunders JB, Aasland OG, Babor TF, De la Fuente JR, Grant M. Development of the Alcohol Use Disorders Identification Test (AUDIT): WHO Collaborative Project on Early Detection of Persons with Harmful Alcohol Consumption-II. Addiction. 1993 Jun;88(6):791-804.

34. Heishman SJ, Singleton EG, Liguori A. Marijuana Craving Questionnaire: Development and initial validation of a self-report instrument. Addiction. 2001;96(7):1023-34.

35. Hammes JGW. De Stroop Kleur-Woord Test. Handleiding. Lisse: Swets and Zeitlinger; 1971.

36. Scarpina F, Tagini S. The stroop color and word test. Frontiers in Psychology. 2017;8:1-8.

37. JASP Team. JASP (Version 0.14.1). 2020. 
38. R Core Team. R: A language and environment for statistical computing. Vienna: R Foundation for Statistical Computing; 2013.

39. Moon K-W. processR: Implementation of the "PROCESS" Macro. 2021.

40. Rosseel Y. lavaan: an R package for Structural Equation Modeling. Journal of Statistical Software. 2012;48(2):1-36.

41. Field M, Eastwood B. Experimental manipulation of attentional bias increases the motivation to drink alcohol. Psychopharmacology. 2005;183(3):350-7.

42. Hester R, Dixon V, Garavan H. A consistent attentional bias for drug-related material in active cocaine users across word and picture versions of the emotional Stroop task. Drug and Alcohol Dependence. 2006;81(3):251-7.

43. Field M, Christiansen P, Cole J, Goudie A. Delay discounting and the alcohol Stroop in heavy drinking adolescents. Addiction. 2007;102(4):579-86.

44. Christiansen P, Schoenmakers TM, Field M. Less than meets the eye: Reappraising the clinical relevance of attentional bias in addiction. Addictive Behaviors. 2015;44:43-50.

45. Marissen MAE, Franken IHA, Waters AJ, Blanken P, van den Brink W, Hendriks VM. Attentional bias predicts heroin relapse following treatment. Addiction. 2006;101(9):130612.

46. Carpenter KM, Martinez D, Vadhan NP, Barnes-Holmes D, Nunes E v. Measures of attentional bias and relational responding are associated with behavioral treatment outcome for cocaine dependence. American Journal of Drug and Alcohol Abuse. 2012;38(2):146-54.

47. Cox WM, Hogan LM, Kristian MR, Race JH. Alcohol attentional bias as a predictor of alcohol abusers' treatment outcome. Drug and Alcohol Dependence. 2002;68(3):237-43. 
48. Spiegelhalder K, Jähne A, Kyle SD, Beil M, Doll C, Feige B, et al. Is smoking-related attentional bias a useful marker for treatment effects? Behavioral Medicine. 2011;37(1):26-34.

49. UNODC. World Drug Report 2016. 2016.

50. Hooker WD, Jones RT. Increased susceptibility to memory intrusions and the Stroop interference effect during acute marijuana intoxication. Psychopharmacology. 1987;91(1):204.

51. Crean RD, Crane NA, Mason BJ. An Evidence-Based Review of Acute and Long-Term Effects of Cannabis Use on Executive Cognitive Functions. Journal of Addiction Medicine. 2011 Mar;5(1):1-8. 


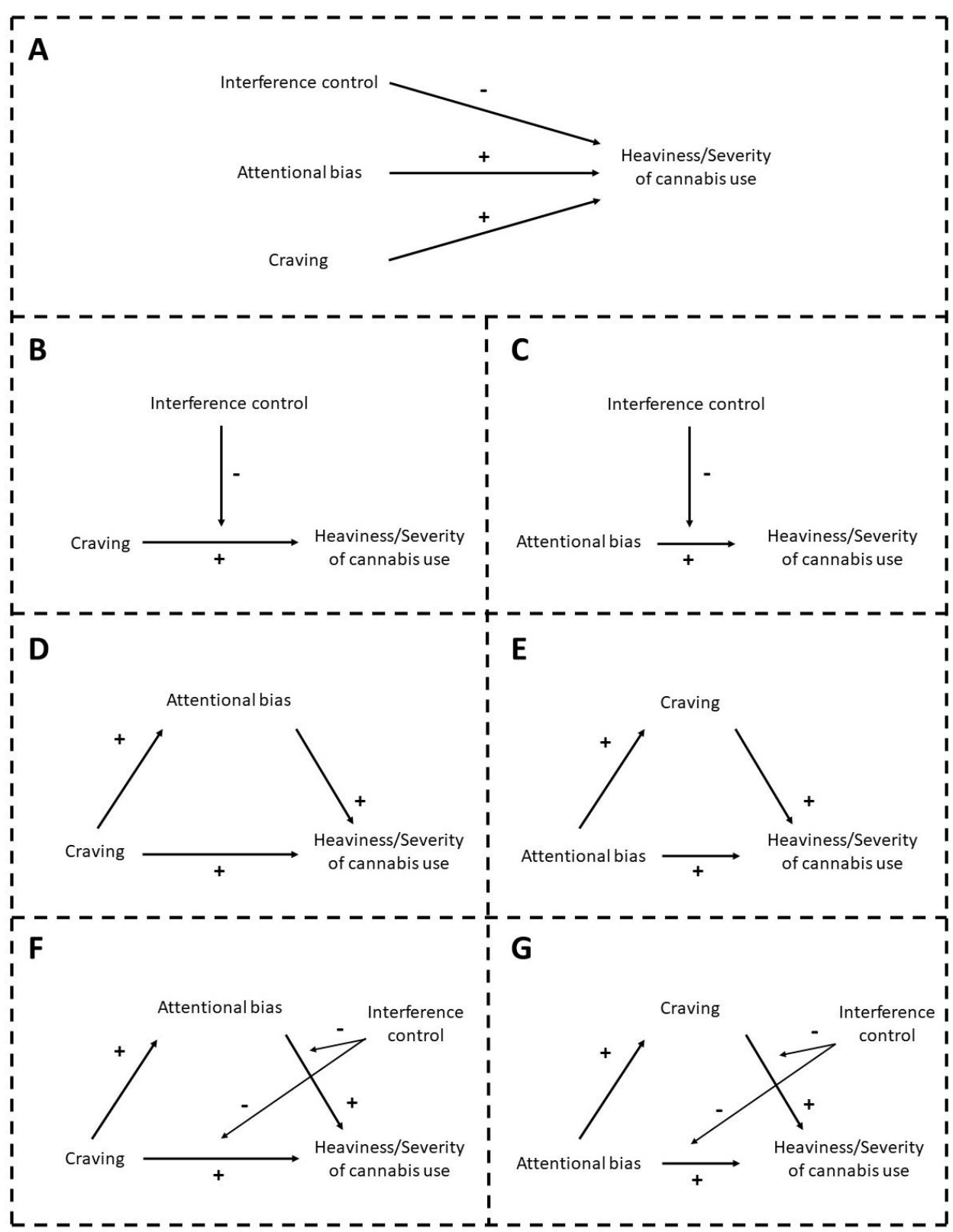

Figure 1. Visual representation of hypotheses. 


\begin{tabular}{|c|c|c|c|c|c|c|c|}
\hline \multirow[b]{2}{*}{ Variables } & \multicolumn{6}{|l|}{ Groups } & \multirow{2}{*}{$\begin{array}{l}\text { Occasional } \\
\& \quad \text { Regular } \\
(\mathrm{N}=358)\end{array}$} \\
\hline & $\begin{array}{l}\text { Never- } \\
\text { Sporadic } \\
(\mathrm{N}=97)\end{array}$ & $\begin{array}{l}\text { Occasional } \\
(\mathrm{N}=35)\end{array}$ & $\begin{array}{l}\text { Regular } \\
(N=323)\end{array}$ & $\begin{array}{l}\text { CUD } \\
(N=97)\end{array}$ & Group Difference & $\begin{array}{l}\text { Pairwise } \\
\text { Difference }^{\#}\end{array}$ & \\
\hline Gender, $\%$ male & 57.7 & 45.7 & 75.9 & 77.7 & $\begin{array}{l}\chi^{2}(3, N=549)=24.9, \\
p<.001\end{array}$ & $2,3,4,5$ & 72.9 \\
\hline Age, $M(S D)$ & $22.7(3.2)$ & $21.9(2.7)$ & $23.3(6.0)$ & $20.6(3.0)$ & $\begin{array}{l}F(3,539)=7.2 \\
p<.001, \eta^{2}=.04\end{array}$ & 3,6 & $23.2(5.8)$ \\
\hline CUDIT-R, M(SD) & - & $2.0(1.5)$ & $15.4(5.2)$ & $21.6(6.6)$ & $\begin{array}{l}F(2,441)=169.0 \\
p<.001, \eta^{2}=.43\end{array}$ & $4,5,6$ & $14.1(6.4)$ \\
\hline Gram/Week, M(SD) & - & $.3(1.2)$ & $4.7(4.0)$ & $9.2(7.9)$ & $\begin{array}{l}F(2,415)=46.0 \\
p<.001, \eta^{2}=.18\end{array}$ & $4,5,6$ & $4.2(4.0)$ \\
\hline Age of onset, $M(S D)$ & - & $16.9(2.3)$ & $15.7(2.3)$ & $15.6(2.2)$ & $\begin{array}{l}F(2,429)=3.7 \\
p<.001, \eta^{2}=.02\end{array}$ & 4,5 & $15.8(2.4)$ \\
\hline Smoking, \% smokers & 19.6 & 40.0 & 64.1 & 85.3 & $\begin{array}{l}\chi^{2}(3, N=550)=97.5 \\
p<.001\end{array}$ & $2,3,4,5,6$ & 61.7 \\
\hline FTND, $M(S D)$ & $1.3(1.8)$ & $1.9(1.7)$ & $2.8(2.3)$ & $4.1(2.2)$ & $\begin{array}{l}F(3,344)=13.6 \\
p<.001, \eta^{2}=.11\end{array}$ & $2,3,5,6$ & $2.7(2.3)$ \\
\hline AUDIT, $M(S D)$ & $5.6(3.3)$ & $6.4(2.7)$ & $8.8(5.6)$ & $9.3(6.9)$ & $\begin{array}{l}F(3,481)=6.5 \\
p<.001, \eta^{2}=.06\end{array}$ & 2,3 & $8.6(5.5)$ \\
\hline Session induced craving, $M(S D)$ & $-.22(.52)$ & $-.00(1.4)$ & $.64(2.4)$ & $-.27(.95)$ & $\begin{array}{l}F(3,528)=8.7 \\
p<.001, \eta^{2}=.05\end{array}$ & 2,6 & $.58(2.3)$ \\
\hline Average session craving, $M(S D)$ & $-.80(.29)$ & $-.71(.55)$ & $.31(1.0)$ & $-.0003(1.0)$ & $\begin{array}{l}F(3,528)=45.1 \\
p<.001, \eta^{2}=.20\end{array}$ & $2,3,4,5,6$ & $.21(1.0)$ \\
\hline Interference control, $M(S D)$ & $27.5(12.9)$ & $26.9(12.8)$ & $32.1(12.3)$ & $34.8(12.2)$ & $\begin{array}{l}F(3,540)=7.3 \\
p<.001, \eta^{2}=.04\end{array}$ & $2,3,5$ & $31.6(12.5)$ \\
\hline \multirow[t]{7}{*}{ Attentional bias, $M(S D)$} & $-.45(3.4)$ & $-.18(2.8)$ & $.33(.95)$ & 1.1(4.4) & \multicolumn{2}{|c|}{$F(3,541)=3.1, p=.026, \eta^{2}=.02$} & $.28(3.4)$ \\
\hline & \multicolumn{4}{|c|}{ Never-sporadic vs. occasional } & \multicolumn{3}{|c|}{$t(130)=.42, p=.673, d=.08$} \\
\hline & \multicolumn{4}{|c|}{ Never-sporadic vs. regular } & \multicolumn{3}{|c|}{$t(415)=1.96, p=.050, d=.23$} \\
\hline & \multicolumn{4}{|c|}{ Never-sporadic vs. CUD } & \multicolumn{3}{|c|}{$t(188)=2.71, p=.007, d=.39$} \\
\hline & \multicolumn{4}{|c|}{ Occasional vs. regular } & \multicolumn{3}{|c|}{$t(353)=.85, p=.398, d=.15$} \\
\hline & \multicolumn{4}{|c|}{ Occasional vs. CUD } & \multicolumn{3}{|c|}{$t(126)=1.59, p=.114, d=.32$} \\
\hline & \multicolumn{4}{|c|}{ Regular vs. CUD } & \multicolumn{3}{|c|}{$t(411)=1.74, p=.084, d=.20$} \\
\hline \multicolumn{8}{|c|}{$\begin{array}{l}\text { Note: AUDIT: alcohol use disorder identification test; CUD: cannabis use disorder; CUDIT-R: cannabis use disorder identification test; FTND: Fagerström } \\
\text { test for nicotine dependence; } M=\text { mean, SD = standard deviation; " Pairwise differences }(p<.05) \text { after Bonferroni correction; Pairwise comparisons: } 1= \\
\text { never-sporadic vs. occasional, } 2=\text { never-sporadic vs. regular, } 3=\text { never-sporadic vs. CUD, } 4=\text { occasional vs. regular, } 5=\text { occasional vs. CUD, } 6=\text { regular vs. } \\
\text { CUD; }\end{array}$} \\
\hline
\end{tabular}




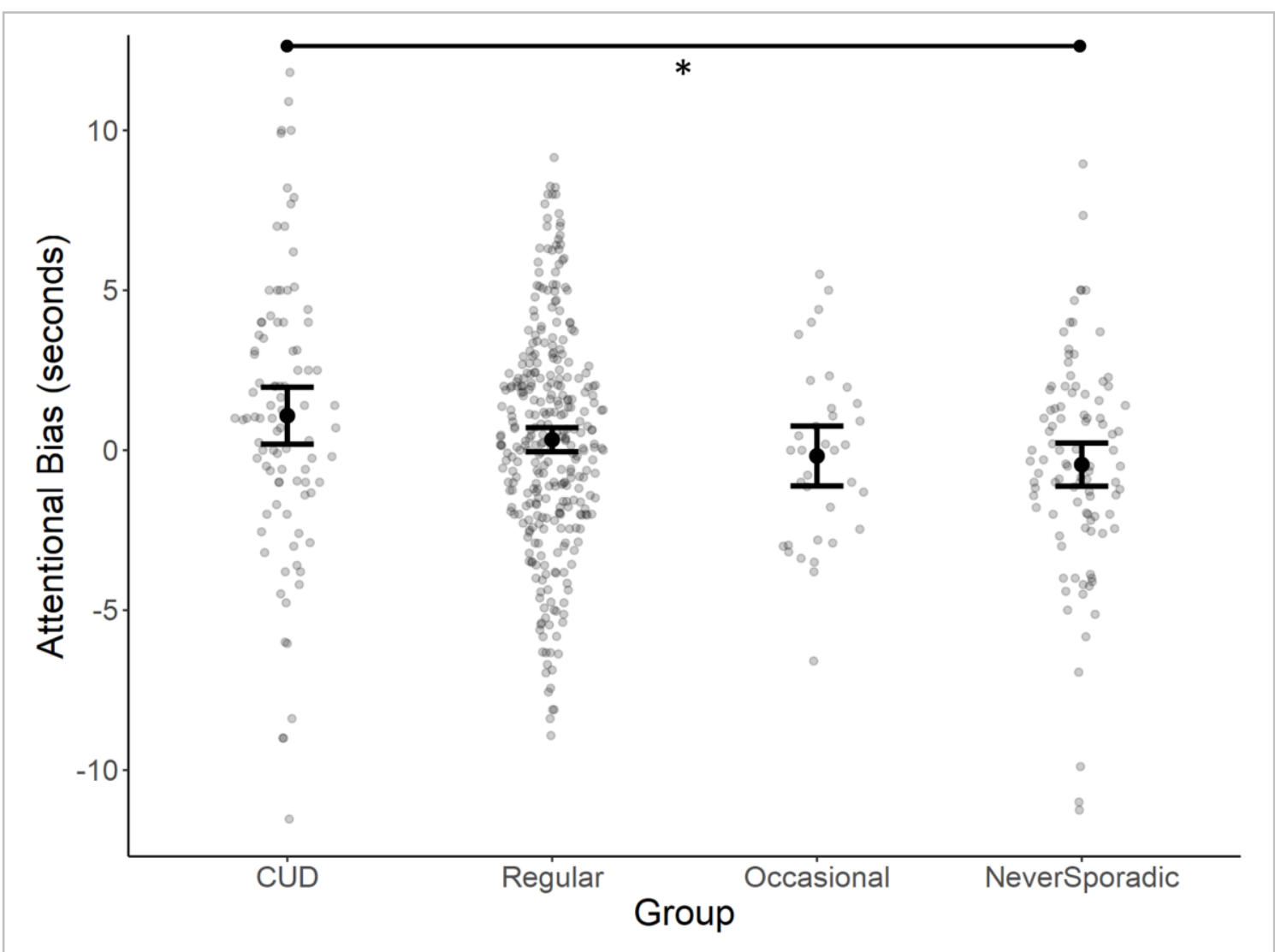

Figure 2. Group differences in attentional bias (AB). Error bars presenting standard error (SE) of the mean. 


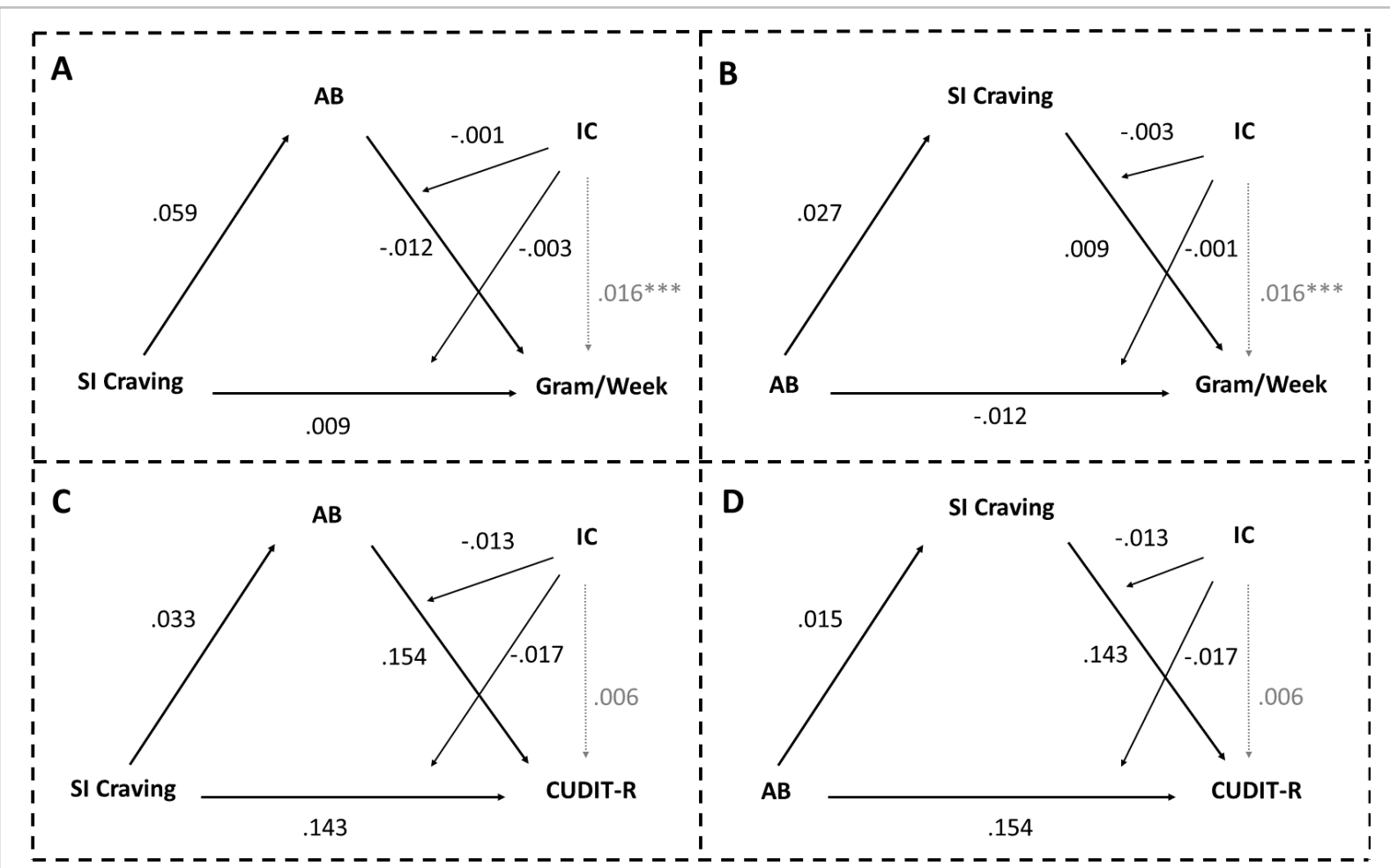

Figure 3. Moderated-mediation analysis results. Analyses assessing the conditional indirect effects of session induced (SI) craving/ attentional bias (AB) on heaviness or severity of use through $A B / S I$ craving, at different levels of interference control (IC). Estimates for all paths reported with indicators of significance: ${ }^{* * *} p<.001$. 


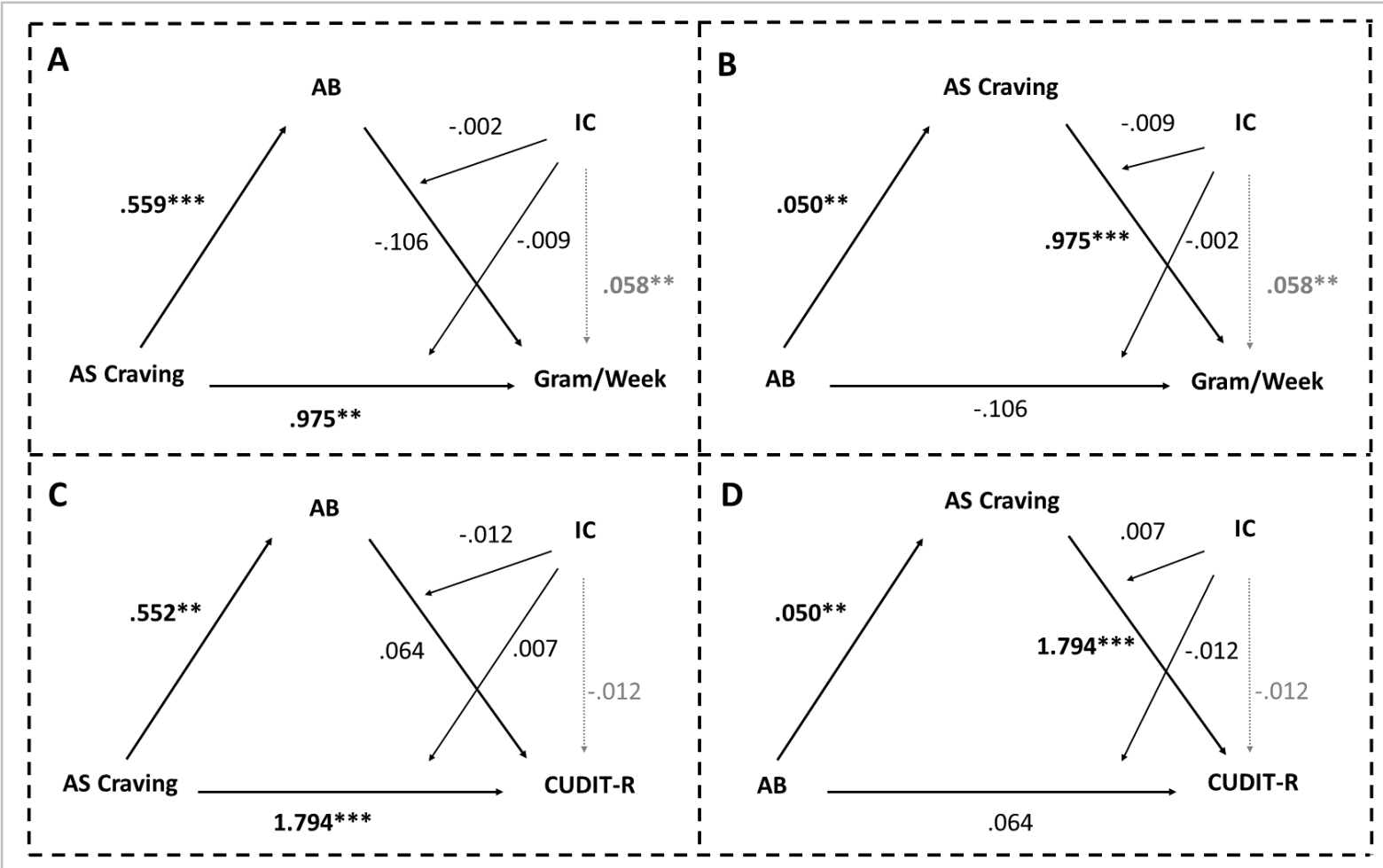

Figure 4. Exploratory moderated-mediation analysis results including average session (AS) craving instead of session induced (SI) craving. Analyses assessing conditional indirect effects of AS craving/attentional bias (AB) on heaviness or severity of use through $A B / A S$ craving, at different levels of interference control (IC). Estimates for all paths reported with indicators of significance: ${ }^{* *} p<.01 * * * p<.001$. 